\title{
FAST DETECTION OF NUMBER OF ANTENNA PORTS IN LTE SYSTEM
}

\author{
Sajal Kumar Das ${ }^{1}$, Om Prakash ${ }^{2}$ \\ ${ }^{1}$ Ericsson R\&D, Bangalore, KA, India \\ ${ }^{2}$ JJT University, Jhunjhunu, Rajasthan, India
}

\begin{abstract}
In LTE system, during initial cell selection UE is unaware about the number of antennas used by eNB for transmission. So, UE blindly tries multiple times to detect the right number of antennas used for transmission in the system. This wastes lot of time and UE processing power, as UE needs to do channel estimation, equalization/demodulation, decoding process multiple times with assumption of 1 or 2 and 4 antenna ports each time.
\end{abstract}

The objective of this paper is to find out a faster and efficient method for detecting the number of antenna ports used by the eNB for signal transmission. A new method is explored for detecting the number of eNB transmit antennas before starting PBCH decoding and CRC checking by exploiting the presence of downlink reference signals at various Resource Element (RE) positions in the Resource Blocks (RB) and using the PBCH SFBC data patterns. This helps for faster detection of number of antennas used for transmission that in turn helps to reduce the UE power consumption as well as reduces the initial cell search time.

Keywords: UE- User Equipment, LTE-Long-Term Evolution, eNB- evolved Node B, RAT-Radio Access Technology, PBCH-Physical Broadcast Channel, SFBC-Space Frequency Block Codes, DL-Down Link.

\section{INTRODUCTION}

To satisfy the ever increasing demand for higher data rate and running complex data voracious applications, the current cellular system is evolving towards next generation cellular systems commonly known as Long-Term Evolution (LTE). It is a 3GPP standard for wireless cellular communication for achieving high-speed data for mobile phones and data terminals.

In cellular systems the Broadcast Channel (BCH) is transmitted periodically which contains the basic system information that allows the UE to operate and configure other channels. In LTE system, the part of the $\mathrm{BCH}$ transport channel which carries Master Information Block (MIB) is mapped to physical broadcast channel (PBCH). The MIB consists of a limited number of the most frequently transmitted parameters essential by UE for initial access to the cell. PBCH is transmitted over four sub-frames with $40 \mathrm{~ms}$ timing interval and always transmitted with a fixed bandwidth. The detectability of this bandwidth by UE without having prior knowledge of the system bandwidth is achieved by mapping the $\mathrm{PBCH}$ only to the central 72 subcarriers of the OFDM signal. That means $\mathrm{PBCH}$ is always transmitted with central $72 * 15 \mathrm{KHz}$ central bandwidth, regardless of the total transmitted system bandwidth. UE first identifies the system center-frequency (dc) from the synchronization signals during the cell search procedure and then reads the $\mathrm{PBCH}$ information accordingly. The $\mathrm{PBCH}$ transmission processing steps are depicted in Fig-1.

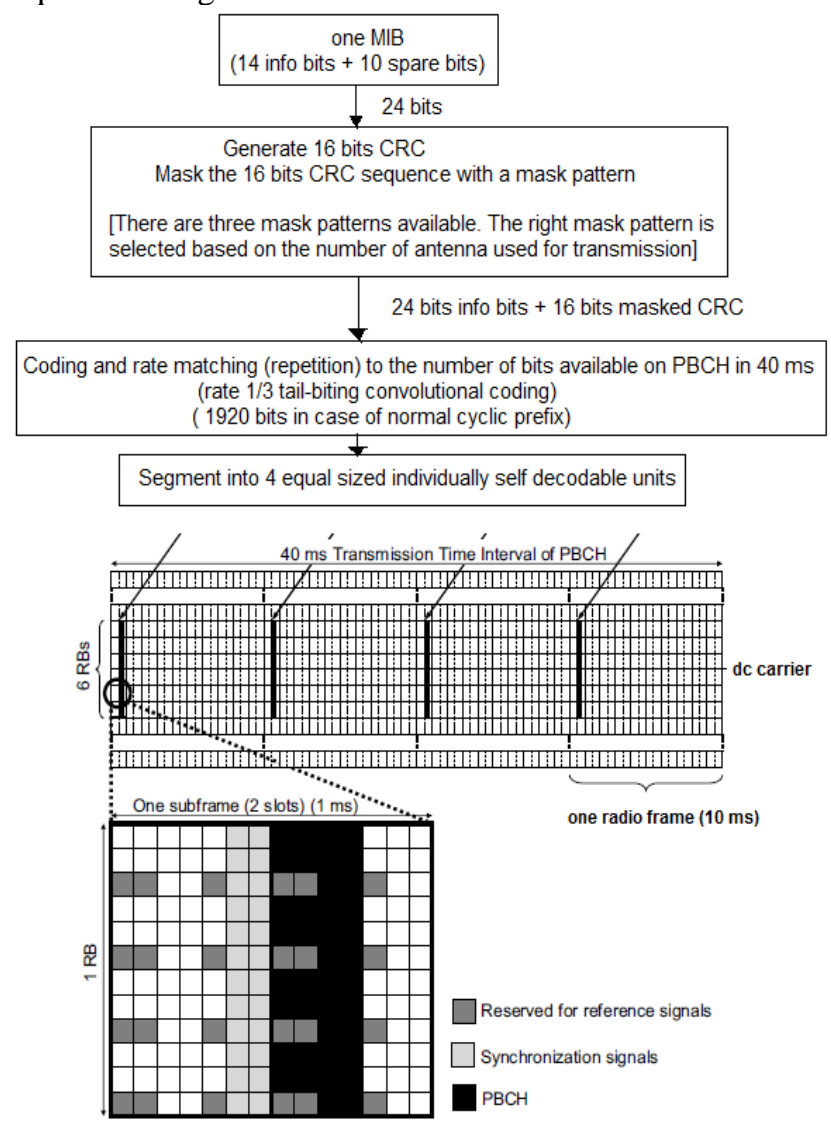

Fig-1, Transmission of PBCH in LTE system 
The size of the MIB is 14 bits and it contains- (a) DL Bandwidth (3 bits) - it indicates the System bandwidth used in the cell (1.4MHz ...20 MHz), (b) PHICH Configuration (3 bits), (c) System Frame Number (8 bits), (d) Spare bits (10 bits). But the number of antennas used by eNB is not communicated directly to UE, rather it is indirectly communicated through CRC masking. The CRC of the transmitted MIB data undergoes through a special mask to encode this information. On the UE receiver side, the UE tries several times iteratively to determine the applied mask to detect the antenna configuration.

For physical layer processing, the $\mathrm{BCH}$ data arrives to the encoding unit in the form of a maximum of one transport block every transmission time interval (TTI) of $40 \mathrm{~ms}$. As discussed earlier, in $\mathrm{PBCH}$ there are 14 information bits and 10 spare bits, so total 24 bits. Then from this information bits the CRC parity bits are computed. The eNB can use 1, 2 or 4 antennas for transmission. On the eNB transmission side, the CRC bits are scrambled (masked) according to the mask pattern corresponding to 1 , 2 or 4 antenna. The scrambling is performed according to the eNode-B transmit antenna configuration with the sequence $\mathrm{X}_{\mathrm{ant}, \mathrm{k}}$ as indicated in Table 1 to form the sequence of bits $c_{k}$ as below [1]:

$$
c_{k}=\left\{\begin{array}{cc}
a_{k} & 0 \leq k<A \\
\left(p_{k-A}+x_{\text {ant }, k-A}\right) \bmod 2 & A \leq k<(A+L) .
\end{array}\right.
$$

Where, $\mathrm{L}=16$.

Table-1

\begin{tabular}{ll}
\hline $\begin{array}{l}\text { Number of transmit antenna } \\
\text { ports at eNode-B }\end{array}$ & PBCH CRC mask $\left\langle x_{\text {ant }, 0}, x_{\text {ant }, 1}, \ldots, x_{\text {ant, } 15}\right\rangle$ \\
\hline 1 & $\langle 0,0,0,0,0,0,0,0,0,0,0,0,0,0,0,0\rangle$ \\
2 & $\langle 1,1,1,1,1,1,1,1,1,1,1,1,1,1,1,1\rangle$ \\
4 & $\langle 0,1,0,1,0,1,0,1,0,1,0,1,0,1,0,1\rangle$ \\
\hline \hline
\end{tabular}

As shown in Fig-1, the 16 bits parity bits are generated and appended to data sequence of 24 bits. As shown in Table-1, there are 3 sequences 0x0000, 0x5555 \& $0 x f f f f$ corresponding to 1,2 or 4 eNB antennas. So, total $14+10+16=40$ bits of data generated after CRC addition. Once CRC is attached, the bit sequence $c_{k}$ is encoded using a rate $1 / 3$ tail-biting convolutional coder. Next, the resultant coded bits are rate matched using the circular buffer approach to obtain the rate matched sequence $b(0)$, $\mathrm{b}(1), \ldots, \mathrm{b}\left(\mathrm{N}_{\mathrm{bit}}-1\right)$, where $\mathrm{N}_{\text {bit }}$ is the number of bits transmitted on the $\mathrm{PBCH}$ and it is dependent on the length of cyclic prefix. Then this sequence is scrambled with a cell-specific scrambling sequence and mapped to OFDM time-frequency resource grid and modulated using QPSK modulation, and transmitted. PBCH data is not channel interleaved. In time scale, the $\mathrm{PBCH}$ is transmitted in the first four OFDMA symbols of the 2nd slot of each radio frame in case of FDD mode as shown in Fig-1. A single antenna, two-antenna SFBC and four-antenna SFBC-FSTD transmit diversity schemes are supported on the $\mathrm{PBCH}$.
On the receiver side, UE knows the cell specific scrambling code derived from the cell ID which is obtained from detected PSS and SSS. So, after reception of $\mathrm{PBCH}$ data, it descrambles and decodes the data. The PBCH does not occupy subcarriers reserved for cell specific reference signals of the four antenna ports irrespective of number of antennas used for $\mathrm{PBCH}$ transmission. This is for simplicity reasons because during the initial $\mathrm{PBCH}$ reception $\mathrm{UE}$ is unaware about the number of antennas used for transmission. So, UE actually performs blind detection of the number of antennas used for $\mathrm{PBCH}$ with hypothesis of the single antenna, two antennas SFBC and four antennas.

\section{PROBLEMS WITH THE EXISTING METHOD}

As described in previous section, when UE obtain MIB after descramble of received $\mathrm{PBCH}$, it need to correctly detect the number of transmitting antenna used for transmission. The information about the number of transmitting antenna used in the system is embedded in $\mathrm{CRC}$ mask of PBCH. Until and unless the number of antenna port is known correctly, UE can't do channel estimation and equalization using correct antenna port number.

In presently existing method, UE blindly tries three times to find out the number of antenna ports used in the transmission. It first assumes that single antenna is used and with that assumption, it picks the reference signals from the resource grid and does the channel estimation, equalization and hence decoding. After decoding it gets 24 bits info sequence and 16 bits received $\mathrm{CRCr}$ bits. Then it generates freshly CRCg (16 bits) bits from the received 24 bits info sequence. The received CRCr (16 bits) is XORed with three types of known CRC masks (16 bits) as given in table-1, which generates three 16 bits sequences, CRCrm1, $\mathrm{CRCrm} 2$, and $\mathrm{CRCrm} 3$ respectively as shown in Fig-2. Then CRCg is compared with CRCrm1, CRCrm2, and CRCrm3. If $\mathrm{CRCg}$ matches with any one of the CRCrm, then that indicates the number antenna used. If one antenna is used, then it will match with CRCrm1. But, if different antenna configuration was used, then it will not match with any one of these above. In that case UE has to again repeat the same process assuming the number of antenna is 2 . If that also does not match, then UE has repeat again assuming number of antennas as 4 .

So, if UE tries to decode PBCH assuming 1, 2, 4 antenna ports, UE may experience three times repetitions of the decoding process before completing the process of decoding $\mathrm{PBCH}$ correctly. It will increase the decoding complexity, and will consume lot of time. This is big processing overhead, and hence causes delay in cell camping and increases power consumption. 
The proposed method in this paper tries to minimize this overhead by using an improved efficient fast detection method.

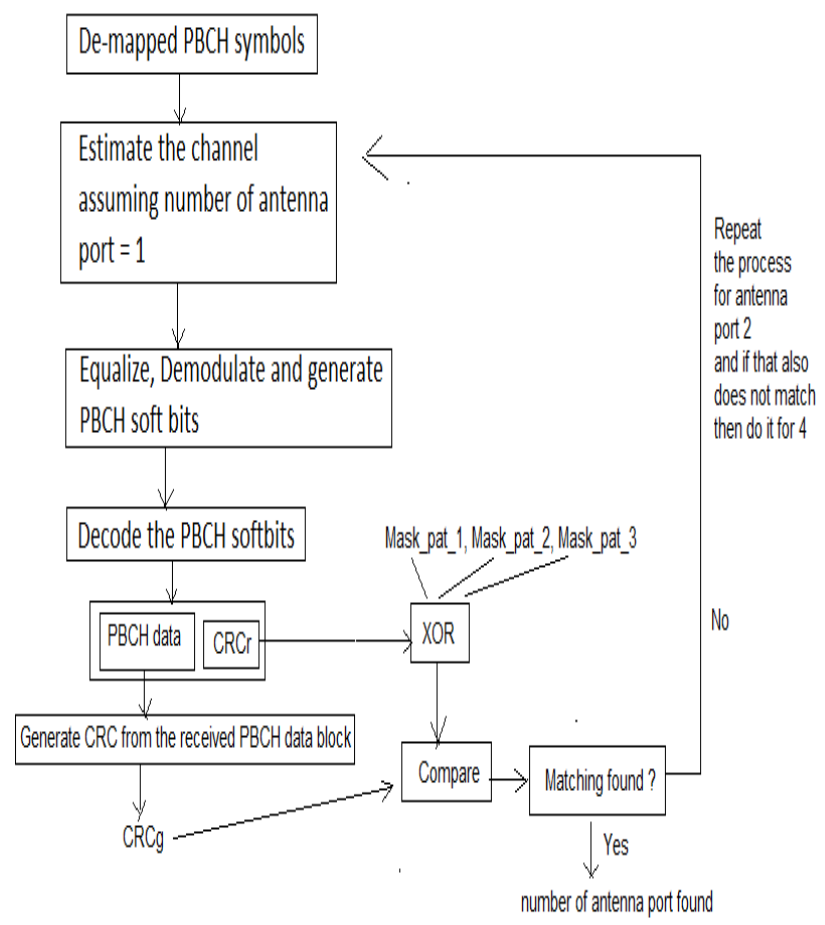

Fig- 2, Legacy method of antenna port detection

\section{IMPROVED METHOD FOR ANTENNA PORT DETECTION}

For faster detection of number of antenna ports and to reduce the amount of calculation and meet the requirements of real-time system, this paper proposes an efficient method of detecting the number of antenna ports of LTE system. The objective of this method is to explore a robust method for detecting number of transmit antennas at the receiver before going for $\mathrm{PBCH}$ decoding and $\mathrm{CRC}$ checking. In this method the following steps will be performed:

(1) Initial cell search and time sync and $\mathrm{PBCH}$ demapping: UE does the initial cell search operation as performed today in the legacy process and does the timing synchronization, and from that it determines the position of PBCH mapping.

(2) Correlation peak estimation for expected reference symbols for different antenna configurations over different RS (downlink reference signal) positions in different REs over the RB: UE needs to perform coherent demodulation for proper channel estimation and for that it uses the DL reference symbols, which are inserted in the OFDM time-frequency grid. As shown in the Fig-3, for single antenna in normal CP mode the DL reference symbols are inserted within the first and third last OFDM symbol of each slot (in time scale) and a frequency domain spacing of six sub-carriers among them. On top of that, there is also a frequency domain staggering of three subcarriers between the first and second reference symbols. In case of two transmit antennas, the reference signals are inserted from both antennas and the reference signals on the second antenna are offset in the frequency domain by three subcarriers. To allow UE to accurately estimate the channel responses, nothing is transmitted on the other antenna at the same time-frequency location of reference signals. The reference symbols are complex values and are determined according to the symbol position as well as ID of the cell. The reference symbol transmission patterns are shown in the Fig-3 for eNB using different antenna ports. 

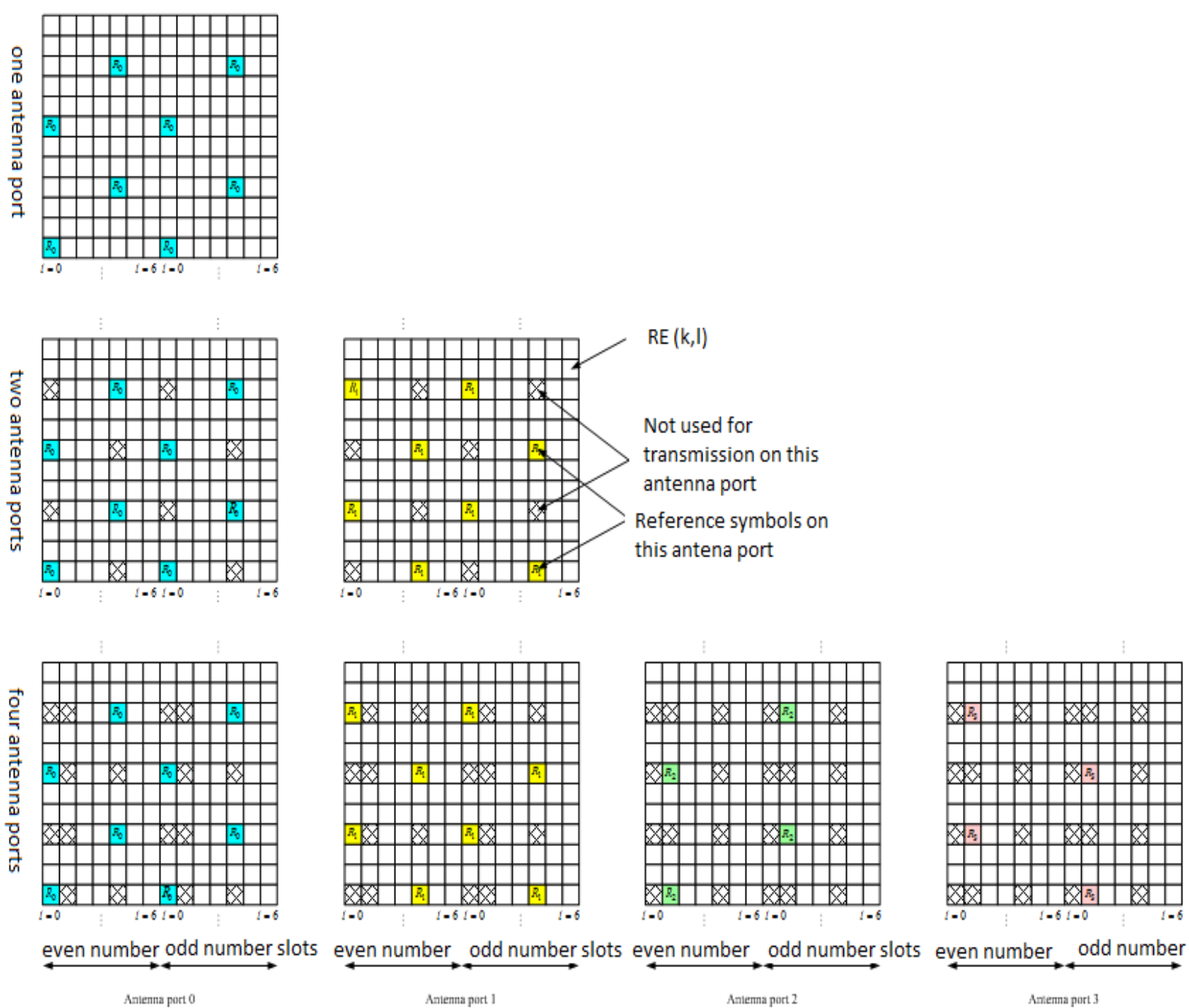

Fig-3, Mapping of downlink reference signals for different antenna ports (normal cyclic prefix)

Assuming that four antenna ports is used for transmission, UE will extract the reference symbols of all the antenna ports in resources of time and frequency domain of $\mathrm{PBCH}$ mapping and these are represented as R0 (cyon colour), R1 (yellow colour), R2 (green colour), and R3(pink colour) in Fig-3. As shown in Fig-4, The PBCH is mapped to the first four symbols in slot \#2 of sub-frame \#1 in the central 6 $\mathrm{RBs}$, but the $\mathrm{PBCH}$ symbols are not present in the resource elements (RE) where downlink Reference symbols are present e.g. it is exclusive of the RE of reference signal under four antenna ports.

First we take 1st symbol position in a slot (say, symbol 1 of slot\#2 over different sub-carriers vertically, as shown in
Fig-4), and extract symbols selectively at the RS locations only. Total, there will be 72 samples for $1^{\text {st }}$ symbol position; out of those, only every third alternative sample will be considered here and selected (assuming more than 1 antenna transmission e.g. 2 or 4). It means we take only reference symbols in 1st slot's 1st symbol positions across 72 sub-carriers. So, we get 24 reference symbol samples from 72 total samples. Again out of 24 samples, the value of odd number of samples is equal to even number of samples. In case of valid two antenna or four antenna ports case, the odd number of samples will have proper symbols e.g. power will be higher at those locations, else for single antenna case these will be null (e.g. hole that means no power is transmitted on those REs). 


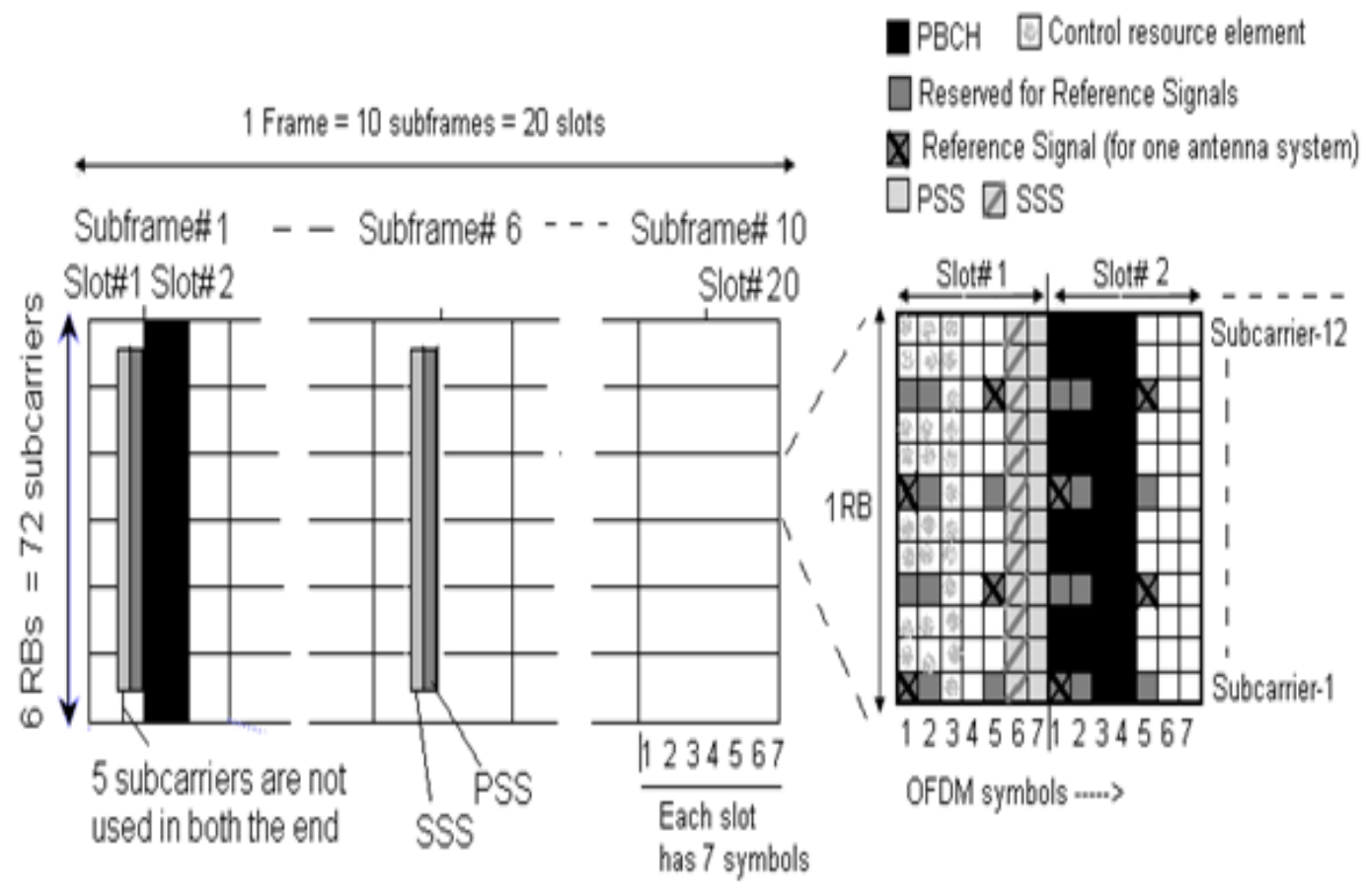

Fig 4, PSS, SSS and PBCH locations in LTE Downlink RBs

The actual RB pattern for multi-antenna case is shown in Fig-3. So, ideally the combined pattern in the space will be as described below:

- In the first symbol of the first slot, subcarrier number- $1,7(=1+6), 13(7+6), 19(13+6), \ldots$, and so on....will contain R0.

- In the first symbol of first slot, subcarrier number $4,10(4+6), 16(10+6), \ldots$ and so on will contain $\mathrm{R} 1$.

- In the second symbol of the first slot, subcarrier number- 1, $7(=1+6), 13(7+6), 19(13+6), \ldots$, and so on....will contain R2.

- In the second symbol of first slot, subcarrier number $-4,10(4+6), 16(10+6), \ldots$. And so on will contain R3.

In case of single antenna case, the locations of R1, R2 and R3 will contain 0 power e.g. null.

In case of two antenna case, the R3 and R4 will be zero or null power.

But, R0 will be always present in the respective REs in RB by default for any antenna configurations (1, or 2 or 4 ). So, sum of power of R0s = SR0 is used as reference to set the threshold for comparison and finding whether any energy is present in R1, R2, R3 resource elements.

Ideally, in case of one antenna port, out of selected 24 samples as above, only even samples will have the values and odd samples will be having zero value (null).
That means as shown in Fig-6, if all R0 samples are extracted from the received data over the RBs and correlate with the locally generated R0 symbols and then sum the values over all the locations then that will provide the sum of all correlated values of R0 energy over different locations. Say, that is SR0. This SR0 will be set as threshold to compare and check whether in R2 and R3 locations contain Null (no power) or respective reference symbols.

So, same way, the R1 symbols are extracted and correlated with locally generated R1 symbols and sum over various R1 locations in the RBs. Say, that sum is SR1.

Now, UE will compare SR1 with SR0. If they are comparable values then it indicates the system is having more than one antenna (as energy is present R1). Otherwise, if R1 is null, it will indicate the system is using only one antenna. In this case, no need to check further for 4 antenna case.

With the above step, only if it is found that the R1 has energy then UE need to check further whether it is 2 or 4 antenna case. To do so UE will extract R2 from various REs in the RBs and compute sum of correlated values of R2 and locally generated R2 over various REs. Say that sum value is SR2.

Compare SR2 with SR0 and if they are comparable then R2 is present that means system is using 4 antennas. Else 
system is using 2 antennas. This will help to detect whether 2 or 4 antenna port is used in the system.

To strengthen the decision further SFBC coding detection could also be used as described next.

(3) SFBC coding detection of PBCH data and verification of earlier derived decision: This method helps to identify further about the correct number of used antenna ports. As discussed earlier, in case of two antenna port transmission, the SFBC is used for $\mathrm{PBCH}$ data transmission as shown in Fig-5.

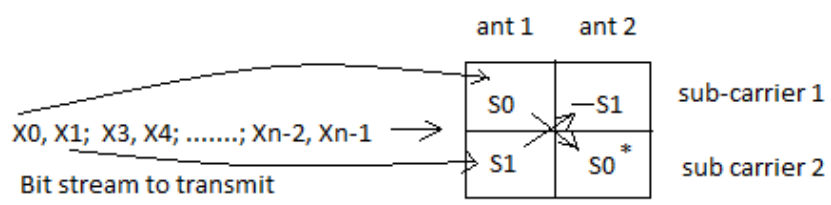

Fig-5, SFBC transmission of $\mathrm{PBCCH}$ transmission using two antennas

So, extract sub-carrier-2, antenna-2 received symbol and make conjugate and then with $\left(\mathrm{S}^{*}\right)^{*}$ correlate the subcarrier-1, antenna-1 received symbol. If the correlation value is high (above a threshold) then that confirms the symbols are SFBC encoded. Similarly perform the same for other positions, like, $\mathrm{S} 1$ and $(-\mathrm{S} 1 *)^{*}$ and so on.

This will further confirm whether the system is using 2 antennas or not.

\section{DECISION MAKING PROCESSING FOR} ANTENNA PORTS:

From step-2 (from detected CRS pattern in the RB) and step-3 (detected SFBC coded data), UE can infer whether the system is using 1,2 or 4 antennas for transmission. Now, this estimation technique will provide the list in order of highest possibility to lowest possibility e.g. 1, 2 or 4 antennas - that means it will indicate which is the most probable one here based on this above algorithm. Then followed by next probable one and then followed by lowest probable one. This will recommend UE to do the $\mathrm{PBCCH}$ decoding in that order, say 2, 4, 1 or like that.

Fig-6 depicts the flow diagram of this complete method.

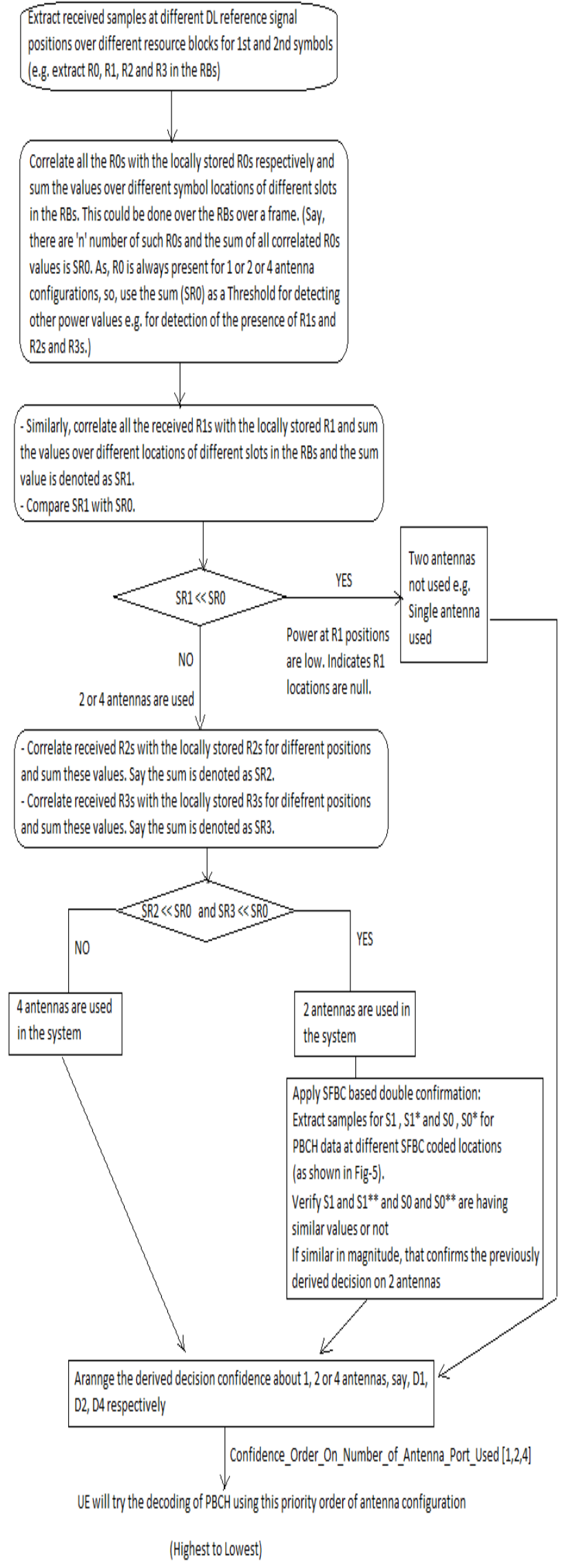

Fig-6, Flow diagram for antenna number selection 


\section{SIMULATION RESULTS}

In simulation as the CRS symbols are pre-known, the locally generated CRS symbols (ideally 12 in numbers) is correlated with received 24 samples (taking even and odd RSes, considering more than one antenna port case as described above). The obtained correlation peaks are compared with a Threshold value (SR0- computed as mentioned above and shown in Fig-6). If we get single correlation peak, then that indicates there could only single antenna used. If we get two peaks that indicate there could 2 or 4 antennas used in the system.

Now, extract the symbols in the same way for next symbol position in that slot e.g. extract the 2 nd symbols in the slot. There will be total 72 symbols here as well. Out of these, as earlier, extract every third samples span over the subcarriers. It will give total 24 samples. There are total 12 even and 12 odd known CRS symbols for those locations. So, we perform correlation between received 12 even samples with the 12 even samples and 12 odd samples with the 12 odd samples. If it gives two identical peaks (more or less same amplitudes) then it indicates the system is using 4 antennas. Else, the system is using 2 antennas. So, the output from this module will be number of antenna ports used in the system.

The results of simulation of this proposed method are shown in Figs. 6-7 for single and two antenna transmissions respectively. The Fig-8 shows percentage of correctly detected antenna port at the first iteration using this proposed method. That indicates, now most of the time $\mathrm{UE}$ is able to detect the right number of antenna port used in the system at the first iteration itself using this proposed method.

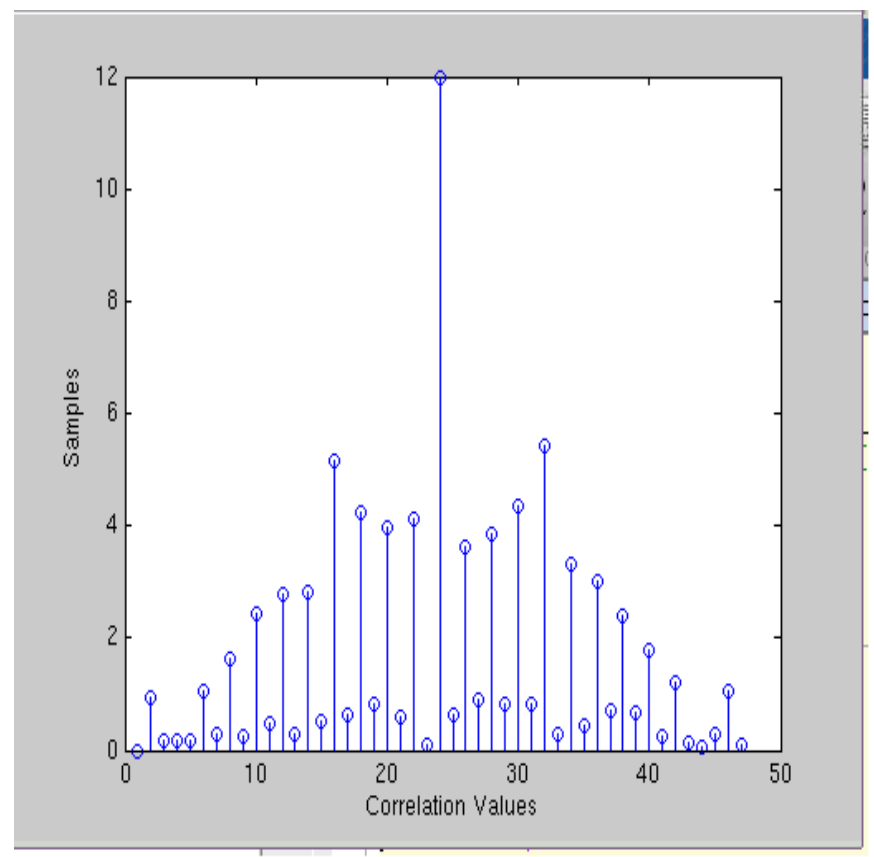

Fig-6, Simulation results for automatic detection of ports (P

$$
=1, \mathrm{SNR}=0 \mathrm{~dB} \text { ) }
$$

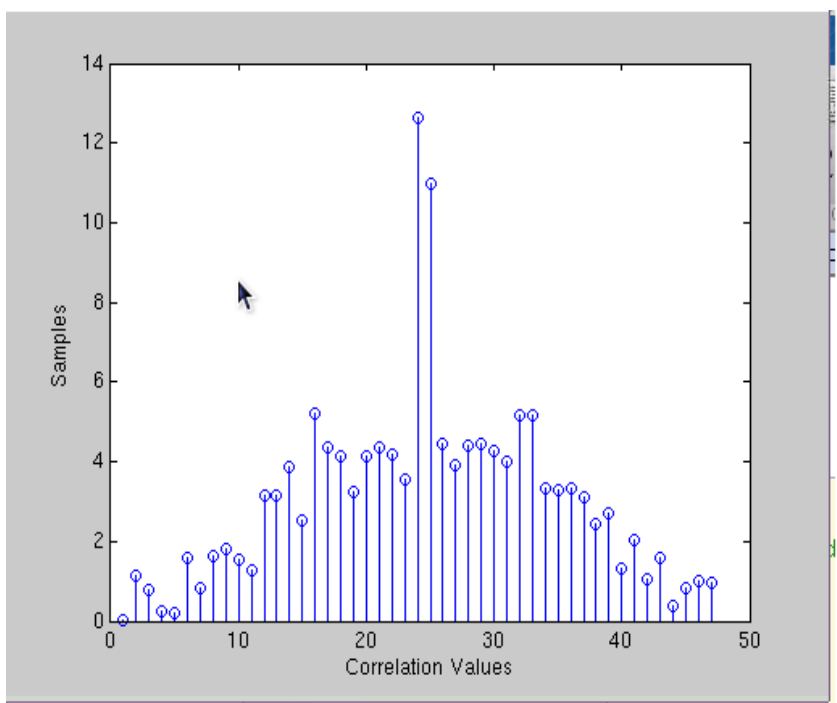

Fig-7, Simulation results for automatic detection of ports (P $=2, \mathrm{SNR}=0 \mathrm{~dB}$ )

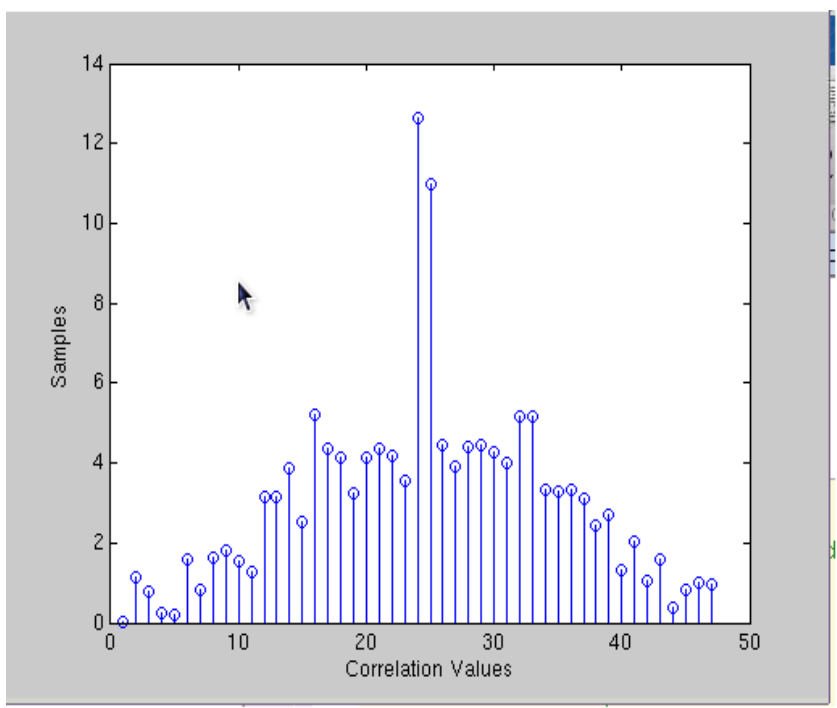

Fig-7, Simulation results for automatic detection of ports $(\mathrm{P}$ $=2, \mathrm{SNR}=0 \mathrm{~dB}$ )

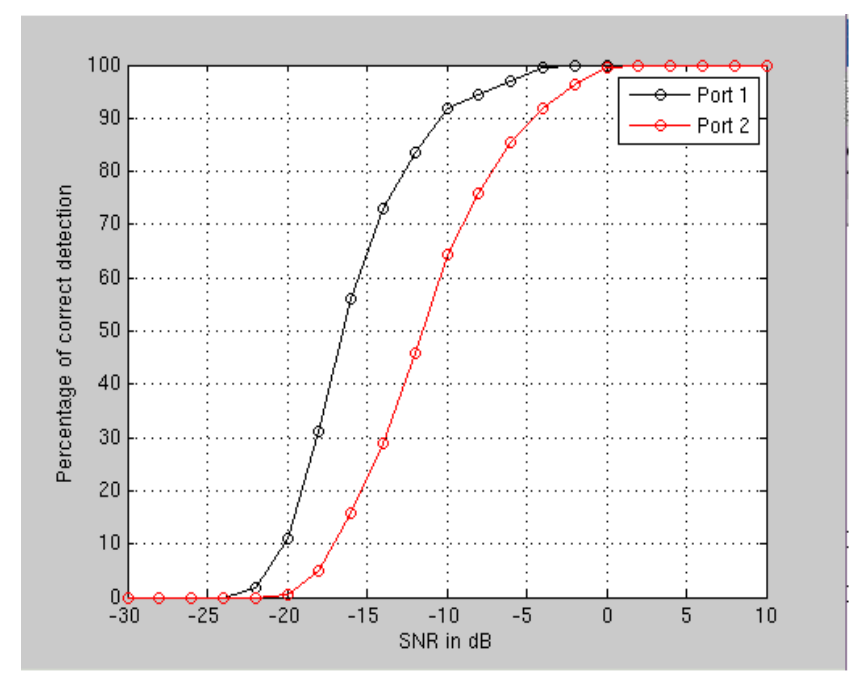

Fig-8, Percentage of correct antenna port detection 


\section{CONCLUSION}

This new proposed method will help significantly to make the $\mathrm{PBCH}$ detection much faster. The system simulation shows that it could be 3:1 times faster than legacy methods. The main advantage of this proposed method is that UE need not have to decode the PBCCH with blind assumption of how many transmit antennas are used at eNB, rather in the first attempt itself it can decode the PBCCH successfully and find out the number of antenna ports used in the system. That will help UE in many ways like, reducing the UE processing power and battery consumption, reducing operation delay and initial cell selection and hence achieving better UE LTE and IRAT performance.

\section{REFERENCES}

[1]. 3GPP TS 36.211 v9.0.0 Evolved Universal Terrestrial Radio Access (E-UTRA) Physical Channels and Modulation (Release 9) [S] 2009-12.

[2]. Mai Vu, Arogyaswami Paulraj, MIMO wireless precoding [J], IEEE signal processing magazine Feb 2006.

[3]. Sajal Kumar Das, "Mobile Handset Design", JOHN WILEY \& SONS, ISBN: 978-0470824672, April 2010. 